\title{
Adoecimento de trabalhadores de enfermagem no contexto hospitalar
}

\author{
Illness among nursing workers in the hospital context \\ Enfermedad de los trabajadores de enfermería en el contexto del hospital
}

\author{
Ana Terra Porciúncula Baptista'; Norma Valéria Dantas de Oliveira Souza"; Cristiane Helena Gallasch"I'; \\ Thereza Christina Mó Y Mó Loureiro Varella"v; Isabela da Rosa Noronhav; Isabele da Rosa Noronha ${ }^{\text {I }}$
}

\begin{abstract}
RESUMO
Objetivo: determinar as questões de saúde que levam os profissionais de enfermagem a licenciarem-se do trabalho por mais de 15 dias ou sofrerem readaptação funcional. Métodos: pesquisa documental, retrospectiva com abordagem quantitativa. Foram analisados 886 registros de afastamentos da equipe de enfermagem de um hospital universitário. Os dados foram apresentados por meio de estatística descritiva. Resultados: os afastamentos dos trabalhadores foram atribuídos, principalmente, transtornos mentais e comportamentais (22,6\%), seguidos das doenças do sistema osteomuscular e do tecido conjuntivo (24,4\%) dos casos. Em relação aos motivos que levaram os profissionais a sofrerem readaptação funcional, captou-se como principal causa as doenças osteomusculares (56,3\%), registrando-se em seguida os transtornos mentais (18,8\%). Conclusão: os trabalhadores possuem elevado índice de adoecimento por doenças osteomusculares, no entanto, preocupa o crescente adoecimento mental. Portanto, é necessário intensificar medidas protetoras para prevenção do adoecimento e melhoradas condições laborais.
\end{abstract}

Descritores: Enfermagem; saúde do trabalhador; licença médica; readaptação ao emprego.

\section{ABSTRACT}

Objective: to determine the health problems that lead nursing professionals to leave work for more than 15 days or to undergo functional readjustment. Methods: in this quantitative, retrospective, documentary study, 886 records of absence of the nursing staff at an university hospital were examined. Data were presented using descriptive statistics. Results: workers' absences were attributed mainly to mental and behavioral disorders (22.6\%), followed by musculoskeletal system and connective tissue diseases (24.4\%). The main causes that led nursing staff to undergo functional rehabilitation, were musculoskeletal diseases (56.3\%), followed by mental disorders (18.8\%). Conclusion: nursing workers display high rates of illness from musculoskeletal diseases; however, growing mental illness is worrisome. Therefore, it is necessary to intensify protective measures to prevent illness and improve working conditions.

Descriptors: Nursing; occupational Health; sick leave; employment, supported.

\section{RESUMEN}

Objetivo: determinar los problemas de salud que llevan a los profesionales de enfermería al alejamiento del trabajo por más de 15 días o a una readaptación funcional. Métodos: investigación documental, retrospectiva con enfoque cuantitativo. Se analizaron 886 registros de alejamientos del equipo de enfermería en un hospital universitario. Los datos se presentaron a través de estadística descriptiva. Resultados: los alejamientos de los trabajadores se atribuyeron principalmente a trastornos mentales y comportamentales $(22,6 \%)$, y a las enfermedades del sistema músculo esquelético y del tejido conjuntivo (24,4\%). Respecto a los motivos que llevaron a los profesionales a pasar por readaptación funcional, las principales causas fueron las enfermedades músculo esqueléticas (56,3\%) y, después, trastornos mentales (18,8\%). Conclusión: los trabajadores tienen una elevada tasa de enfermedades musculo esqueléticas, sin embargo, es preocupante el creciente número de enfermedades mentales. Por lo tanto, es necesario intensificar las medidas de protección para prevenir enfermedades y mejorar las condiciones de trabajo.

Descriptores: Enfermería; salud laboral; ausencia por enfermedad; empleos subvencionados.

\section{INTRODUÇÃO}

Durante a década de 1990, houve a incorporação dos hospitais universitários (HU) ao Sistema Único de Saúde (SUS). Visando viabilizar o processo de forma menos onerosa e ajustada à direção política da Reforma do Estado do Governo Federal, realizou-se ajuste na forca de trabalho dessas instituições que aderiram ao Regime Jurídico Múltiplo (RJM). Tal mudança, possibilitou diferentes formas de vinculação dos trabalhadores, trazendo a flexibilização das relações de trabalho e a escassez de concursos públicos ${ }^{1}$.

'Enfermeira. Residente em Enfermagem Neonatal pelo Instituto Fernandes Figueira (IFF/FIOCRUZ). Rio de Janeiro, Brasil. Brasil. E-mail: anaterrapb@gmail.com "Enfermeira. Doutora em Enfermagem. Professora da Universidade do Estado do Rio de Janeiro, Brasil. E-mail: norval_souza@yahoo.com.br

"'Enfermeira. Doutora em Enfermagem. Professora da Universidade do Estado do Rio de Janeiro, Brasil. E-mail: cristiane.gallasch@gmail.com IVEnfermeira. Doutora em Enfermagem. Professora da Universidade do Estado do Rio de Janeiro, Brasil. E-mail: thereza1208@gmail.com vGraduanda em Enfermagem. Faculdade de enfermagem, Universidade do Estado do Rio de Janeiro, Brasil. E-mail: isabela.rosa@@hotmail.com

V'Graduanda em Enfermagem. Faculdade de enfermagem, Universidade do Estado do Rio de Janeiro, Brasil. E-mail: isabele-rosa@hotmail.com 
A precarização das relações de trabalho, decorrente do modelo neoliberal, baseia-se em preceitos que influenciam diretamente o mundo do trabalho: os trabalhadores possuem vínculos precários, perdendo a estabilidade em seus empregos e os direitos trabaIhistas. Além disso trabalham em jornadas extensas e recebem remunerações ignominiosas, portanto para manter-se em condições mínimas de subsistência expõem-se a jornadas duplas e até triplas de trabalho ${ }^{2}$.

Essas questões refletem de forma adversa na saúde do trabalhador, provocando manifestações como estresse, taquicardia, hipertensão arterial sistêmica, sonolência, sudorese, esgotamento físico e mental, depressão, fadiga, cefaleia, dor epigástrica e irritabilidade ${ }^{3}$. Estas manifestações comprometem a assistência prestada aos pacientes e a qualidade de vida dos profissionais ${ }^{4}$.

Condições inerentes ao trabalho de enfermagem, como o lidar com o sofrimento e a vivência do processo de morte e morrer ${ }^{5}$, somam-se à alta demanda no trabalho, rígida supervisão sobre as atividades desempenhadas, baixos salários e o reduzido reconhecimento social que a profissão detém no cenário nacional ${ }^{6}$. Tais condições potencializam a vulnerabilidade do trabalhador ao adoecimento e o consequente afastamento de suas atividades laborais.

Nesta perspectiva, o presente trabalho possui como objeto de estudo os motivos que levam os trabalhadores de enfermagem a se licenciarem de suas funções laborais ou sofrerem readaptação funcional. Um estudo realizado com 1.509 profissionais de enfermagem revelou que $20,3 \%$ dos absenteísmos foram em decorrência do adoecimento destes trabalhadores ${ }^{7}$. Acrescenta-se que o afastamento do trabalho influencia negativamente a percepção dos trabalhadores sobre sua qualidade de vida e saúde ${ }^{8}$. Demonstrando o papel central que o trabalho ainda exerce na vida do indivíduo.

$O$ afastamento laboral também gera impacto negativo para o cuidado prestado ao usuário, pois resulta em menos profissionais para dar andamento ao processo de trabalho, sobrecarregando os demais integrantes da equipe de enfermagem que permanecem na assistência, sendo necessária a realização de medidas gerenciais para a substituição daquele funcionário, tais quais o remanejamento de profissionais e/ou a contratação de um funcionário substituto ${ }^{9}$. Porém, ainda que ocorra a substituição do profissional afastado, há perda na qualidade da assistência, pois o tempo demandado para que o substituto alcance o mesmo nível de produtividade do funcionário afastado é significativo.

O licenciamento destes trabalhadores de suas funções gerais, portanto, desencadeia sofrimento para o próprio trabalhador e a precarização da assistência. Portanto, considerando a contextualização inicial, o presente estudo teve como objetivo determinar as questões de saúde que levam os profissionais de enfermagem a licenciarem-se do trabalho por mais de 15 dias ou sofrerem readaptação funcional.

\section{REVISÃo DE LITERATURA}

O trabalho em saúde pode ser realizado dentro de qualquer um dos níveis de atenção: primário, secundário, terciário ${ }^{10}$. Porém, o hospital continua sendo o grande empregador dos profissionais de enfermagem, um ambiente complexo devido à multiplicidade de atores envolvidos no planejamento e execução das ações ${ }^{11}$.

Como situações desencadeadoras de sofrimento psicofísico e adoecimento dos trabalhadores de enfermagem em hospitais, aponta-se o relacionamento entre equipe de enfermagem e paciente-familiares, os ritmos laborais intensos e a necessidade de manter a atenção e a concentração para evitar iatrogenias ${ }^{12,13}$, um processo de trabalho marcado pela exposição a riscos ocupacionais ergonômicos, biológicos, químicos e físicos. Verifica-se que este complexo trabalho, por sua especificidade, apresenta situações que podem espoliar a saúde dos trabalhadores.

Acrescenta-se o delinear de um contexto ainda mais adverso para esses trabalhadores por conta das novas configurações do mundo do trabalho, influenciado pelos preceitos neoliberais ${ }^{14}$. Sabe-se que o trabalho nunca é neutro no processo saúde-doença do trabalhador ${ }^{15}$. Esses processos de desgaste desencadeiam processos de somatização físicos e psíquicos e/ou também desordens emocionais que resultam em sinais de angústia, falta de motivação, medo, ansiedade patológica e depressão ${ }^{16}$.

Como medidas de proteção, buscam-se o uso de equipamentos de proteção individual, educação permanente em serviço, além de máquinas e materiais adequados $^{17}$, além de evitar que esse trabalhador seja exposto ao desgaste ou, quando inevitável, reduzir o seu impacto ${ }^{16}$.

Apesar dessas estratégias, da elevada produção científica no campo da Saúde do Trabalhador que demonstram o nexo causal entre o processo de trabalho e o processo de adoecimento, das ações de promoção e proteção da saúde implementadas pela força da lei e da instituição de modelos assistenciais que buscam a recuperação da força de trabalho, constata-se um coletivo profissional cada vez mais adoecido, desestimulado e com salários aviltantes, que repercutem diretamente na qualidade de vida e que pode gerar alta evasão da profissão, em alto percentual de adoecimento, presenteísmo, aposentadorias precoces e até morte dos trabalhadores.

\section{Metodologia}

Trata-se de uma investigação documental, retrospectiva, com abordagem quantitativa, realizada em um hospital universitário de grande porte, situado no município do Rio de Janeiro, operando, durante o período estudado, aquém de sua plena capacidade, com aproximadamente 235 leitos.

Trata-se de um estudo do tipo censo, ou seja, com contagem e a organização racional de informações, ou seja, análise de todos os elementos de uma determinada 
população ${ }^{18}$. Definiu-se como população, neste estudo, todos os profissionais de enfermagem que atuavam no referido hospital, em regime estatutário, entre janeiro e dezembro de 2015 , que, neste período, correspondiam a 324 enfermeiros e 1.078 técnicos de enfermagem, totalizando 1.402 profissionais.

Foram analisados 886 registros disponíveis nos prontuários de atendimento à saúde desses trabalhadores realizados no Departamento de Segurança e Saúde do Trabalhador, sendo selecionados dados referentes a afastamentos superiores a 15 dias e/ou readaptação funcional, configurando uma amostra de 380 profissionais. O período de afastamento foi definido como sendo superior a 15 dias, pois, na instituição, é partir do $16^{\circ}$ dia de afastamento o trabalhador é encaminhado a perícia no Instituto Nacional do Seguro Social, quando em regime celetista.

Para coleta de dados, foi utilizado formulário desenvolvido pela equipe de pesquisadores que continha as seguintes variáveis: idade, sexo, categoria profissional, tipo de vínculo empregatício, local de atuação, motivo do afastamento, período de afastamento, data de início e término da licença, benefício concedido/ desfecho da situação - afastamento, aposentadoria por invalidez, morte ou readaptação funcional.

Os dados coletados foram codificados e tabulados em uma planilha com o uso do software Microsoft Excel $^{\circ} 2013$. A análise foi realizada por meio de estatística descritiva com conversão em frequências absolutas e relativas para as variáveis categóricas.

O estudo respeitou as exigências éticas contidas nas normas nacionais e internacionais regulamentadoras de pesquisas envolvendo seres humanos, tendo sido aprovado pelo comitê de ética em pesquisa, sob protocolo número 1.690 .589 em 23 de agosto de 2016.

\section{RESULTADOS}

A maioria dos profissionais afastados, no período estudado, pertencia ao sexo feminino (91,3\%), possuía entre 46 e 55 anos $(42,1 \%)$ e trabalhava na instituição como técnico de enfermagem (76,5\%). Cabe destacar que os participantes foram contabilizados por vínculo profissional, ou seja, um indivíduo que porventura possuía mais de uma matrícula no hospital foi contabilizado duas vezes, pois se entende que, apesar de ser um único trabalhador, o impacto do seu afastamento ou readaptação funcional será proporcional ao número de vínculos.

Um estudo realizado no Brasil, cujo objetivo foi traçar o perfil dos profissionais de enfermagem, observou que, apesar do crescimento ocorrido nos últimos anos, de profissionais do sexo masculino, o predomínio ainda é de profissionais do sexo feminino, representando $85,1 \%$ dentre os 1.804 .535 participantes da pesquisa ${ }^{19}$. Nesta perspectiva, o presente trabalho apresenta resultado aproximado a outros estudos ${ }^{20-22}$. As mulheres possuem uma carga de trabalho maior em relação aos homens. Esta carga de trabalho decorre da dupla ou tripla jornada exercida por elas. Além do trabalho profissional (formal), é acrescido também o trabalho doméstico e o cuidado com a família, o que implica um maior desgaste físico e mental dessas profissionais, gerando culpa pelo cumprimento insatisfatório em seus papéis além do profissional, podendo ser traduzido como fator em potencial para o seu adoecimento ${ }^{23}$.

Houve uma grande variedade de motivos que levaram estes profissionais a serem afastados, como observado na Tabela 1. Cabe salientar que o número de afastamentos foi de 829 , porém o quantitativo de motivos encontrados (1.061) é superior, visto que, em alguns casos, os profissionais apresentavam mais de um acometimento que o justificasse. Os dados foram classificados usando o agrupamento em grandes grupos do Código Internacional de Doenças (CID-10).

De acordo com o levantamento, os profissionais foram afastados, majoritariamente, em decorrência de fatores que influenciam o estado de saúde e o contato com serviços de saúde, 261 vezes (24,6\%). Em seguida estavam as doenças do sistema osteomuscular e do tecido conjuntivo, 259 vezes $(24,4 \%)$, e os transtornos mentais e comportamentais, 240 vezes $(22,6 \%)$. Os trabalhadores se afastaram em média 36 dias aproximadamente. Não foram encontrados registros de morte ou invalidez dos profissionais de enfermagem.

Aproximadamente $67,8 \%$ dos profissionais de enfermagem encontram-se entre 31 e 55 anos $^{19}$, desta forma, pode-se verificar que a faixa etária que mais possui afastamentos também é a que apresenta a maior parcela dos profissionais, o que pode demonstrar um viés do estudo. Além disso, já é apontado absenteísmo-doença precoce destes profissionais, sendo mais frequente em profissionais entre 30 e 49 anos $^{21,22}$. 0 adoecimento precoce destes trabalhadores, além de resultar em absenteísmo, também leva à incapacidade de exercer seu trabalho temporária ou permanentemente, comprometendo a assistência e a qualidade de vida do trabalhador ${ }^{4}$.

Em vigência do modelo neoliberal, há a incerteza da permanência no trabalho, elevado desemprego, vínculos laborais informais e instáveis, os quais levam os trabalhadores a permanecerem em mais de um emprego a fim de garantir a subsistência material e um futuro mais estável economicamente. Esta situação reforça o desgaste e o sofrimento psicofísico dos trabalhadores, tendo, assim, potencial ainda maior para o adoecimento do coletivo profissional ${ }^{3}$.

Estudo realizado anteriormente em instituição universitária demonstrou que profissionais de enfermagem se afastam do trabalho por mais tempo e em maior frequência quando comparados a outras categorias profissionais. Verificou-se maior frequência de diagnósticos entre esta população de transtornos de humor, de prescrições de medicação controlada, de 
TABELA 1: Motivos de afastamento apresentados pelos profissionais. Rio de Janeiro, Brasil, 2016.

\begin{tabular}{lc}
\hline \multicolumn{1}{c}{ Motivo de afastamento } & $\mathbf{n}(\%)$ \\
\hline Fatores que influenciam o estado de saúde e o contato com serviços de saúde & $261(24,6)$ \\
Doenças do sistema osteomuscular e do tecido conjuntivo & $259(24,4)$ \\
Transtornos mentais e comportamentais & $240(22,6)$ \\
Lesões, envenenamento e algumas outras consequências de causas externas & $71(6,7)$ \\
Neoplasias [tumores] & $49(4,6)$ \\
Gravidez, parto e puerpério & $45(4,2)$ \\
Doenças do aparelho circulatório & $28(2,6)$ \\
Doenças do sistema nervoso & $27(2,5)$ \\
Sintomas, sinais e achados anormais de exames clínicos e laboratoriais & $16(1,5)$ \\
Doenças do aparelho digestivo & $12(1,1)$ \\
Doenças endócrinas, nutricionais e metabólicas & $11(1,0)$ \\
Doenças do olho e anexos & $9(0,8)$ \\
Algumas doenças infecciosas e parasitárias & $8(0,8)$ \\
Malformações congênitas, deformidades e anomalias cromossômicas & $6(0,6)$ \\
Doenças do sangue e dos órgãos hematopoiéticos e alguns transtornos que comprometem o mecanismo imunitário & $5(0,5)$ \\
Doenças do aparelho gênito-urinário & $5(0,5)$ \\
Doenças do ouvido e da apófise mastoide & $4(0,4)$ \\
Doenças do aparelho respiratório & $2(0,2)$ \\
Doenças da pele e do tecido subcutâneo & $2(0,2)$ \\
Causas externas de morbidade e mortalidade & $1(0,1)$ \\
\hline
\end{tabular}

busca por atendimento da equipe multiprofissional e de afastamentos por transtornos mentais ${ }^{24}$.

Os profissionais desta classe desempenham papel o qual lida diretamente com o sofrimento humano. Acrescenta-se a natureza do trabalho de enfermagem na qual o trabalhador possui baixo controle sobre a atividade desempenhada frente ao alto grau de exigência. Características essas que são notoriamente preditoras do sofrimento psíquico ${ }^{25,26}$.

Constatou-se que a classe que apresentou o maior quantitativo de absenteísmo-doença, neste estudo, foi a dos técnicos de enfermagem, representando (76,6\%). Os técnicos e auxiliares de enfermagem contabilizam atualmente $(77,0 \%)$ da categoria profissional, sendo, portanto, a maior força de trabalho da enfermagem, o que facilmente explicaria a maior incidência de afastamento entre estas categorias ${ }^{19}$. Tais profissionais desempenham atividades que demandam maior esforço físico e elevado desgaste emocional, por estarem em contato direto com os pacientes, vivenciando de perto o processo de adoecimento, sofrimento dos familiares e o processo de morte-morrer ${ }^{5}$, situações que levam ao adoecimento psicofísico, além de estarem mais vulneráveis aos riscos ocupacionais e aos acidentes de trabalho ${ }^{27}$.

Como reflexo da baixa remuneração, os profissionais precisam ter mais de um vínculo empregatício, a fim de suprir suas necessidades pessoais. A multiplicidade de vínculos desgasta os profissionais física e emocionalmente, aumentando a probabilidade de adoecerem. Uma carga horária inferior aliada a uma remuneração adequada tornaria o profissional mais produtivo, com menor risco de adoecimento, consequentemente, afastando-se menos, impactando de forma positiva no custo final do trabalho ${ }^{28}$.
Quanto às causas recorrentes de afastamento apontadas pelo estudo, outros trabalhos com a mesma temática apontam as doenças do sistema osteomuscular e do tecido conjuntivo como a maior causa de afastamento ${ }^{20-22}$. Profissionais de enfermagem são os que mais sofrem com os "Distúrbios Osteomusculares Relacionados ao Trabalho", sendo que as causas referidas pelos próprios profissionais para tal acometimento são: trabalhar por longos períodos na mesma posição, trabaIhar em posições inadequadas e realizar repetidas vezes a mesma tarefa ${ }^{29}$. Como dificilmente poder-se-á mudar a natureza do trabalho da enfermagem, apontada como preditora do adoecimento, é inegável a importância de se reduzir a carga horária destes profissionais.

Em diversas pesquisas, os transtornos mentais e comportamentais foram descritos como uma das cinco primeiras causas de afastamento ${ }^{20-22}$. Em uma série temporal realizada em um hospital universitário, verificou-se que há um aumento em média de $1 \%$ a cada ano de profissionais de enfermagem que se afastam por causa de transtornos mentais e comportamentais ${ }^{30}$. São apontados como principais motivos destes afastamentos os episódios depressivos, o transtorno depressivo recorrente e o transtorno afetivo bipolar ${ }^{5}$.

$\mathrm{Na}$ atualidade, o adoecimento do trabalhador por questões relacionadas à saúde mental é crescente e preocupante. Este fenômeno pode ter relação com o predomínio dos preceitos neoliberais que trazem incertezas ao trabalhador, imposição aos trabalhadores por polivalência e multifuncionalidade, sujeição às pressões do cotidiano laboral, ao vínculo precário de trabalho e a salários cada vez mais aviltantes, entre outros desdobramentos que impactam negativamente a dimensão 
subjetiva do trabalhador. Neste sentido, tem-se um contexto profícuo para desencadear doenças mentais e alterações comportamentais ${ }^{31}$.

Os locais que mais apresentaram profissionais afastados foram: Complexo Cirúrgico com 273 (32,9\%), Cuidado Intensivo com 177 (21,4\%) e os Serviços de atendimento à Mulher e Criança com 148 (17,9\%) afastamentos. Elucida-se que os locais de atuação que possuíam características semelhantes foram agrupados e dispostos em categorias a fim de não haver dispersão de resultados.

Destaca-se o número de dias de afastamento. No total, os afastamentos somaram 29.931 dias de trabalho perdidos, durante o período estudado, um valor bastante expressivo. Este valor é equivalente a aproximadamente 82 anos de trabalho, o que reflete grande prejuízo ao empregador. É reconhecido que esse cenário não apenas gera impacto econômico, como também leva à sobrecarga dos trabalhadores que permanecem no ambiente de trabalho e precisam executar as tarefas dos ausentes ${ }^{32}$.

No que tange aos dados referentes às readaptações, foi encontrado que, análogo ao perfil dos profissionais que sofreram afastamentos, encontrou-se também que a maioria dos profissionais readaptados pertencia ao sexo feminino (79,5\%), possuía entre 46 e 55 anos (70,4\%) e exercia a função de técnico de enfermagem (79,5\%). Ressalta-se o significativo número de enfermeiros que sofreram readaptação funcional, nove profissionais, representando $(20,4 \%)$ da amostra.

Foram encontrados 64 registros de readaptação profissional no período estudado. É importante esclarecer que o quantitativo "Motivo da Readaptação" é superior ao número de profissionais que foram readaptados, pois, em alguns casos, há mais de um motivo que levou o profissional à readaptação funcional.

Para a apresentação de dados foi utilizado o sistema de classificação adotado pelo serviço em que foi realizado o estudo. Esta classificação é semelhante aos grandes grupos do CID-10 e separa os problemas de saúde nas seguintes categorias: osteomusculares, saúde mental, neurológico, gênito-urinário, gastroenterologia, endócrino, auditivo, cardiovascular, respiratório, infecções gerais, hematológico, pele e tecido cutâneo, oftalmológico, câncer e outros. Porém, entre as categorias existentes, a gastroenterologia, as infecções gerais e a hematologia não obtiveram entradas e por isso não são apresentadas na tabela.

Consonante aos motivos de afastamento, as categorias que mais resultaram em readaptação funcional foram: osteomusculares com 36 (56,3\%) ocorrências, seguido por saúde mental com $12(18,8 \%)$ e causas oftalmológicas com 5 ocorrências (7,8\%).

Os locais que mais apresentaram profissionais que sofreram readaptação funcional foram o Complexo Cirúrgico com 14 (26,9\%), a Clínica Médica com 12 $(23,0 \%)$ e o Cuidado Intensivo com 10 (19,2\%), havendo
TABELA 2: Motivos que levaram os profissionais de enfermagem à readaptação. Rio de Janeiro, Brasil, 2016.

\begin{tabular}{lc}
\hline Motivo da Readaptação & $\mathbf{n}(\%)$ \\
\hline Osteomusculares & $36(56,3)$ \\
Saúde Mental & $12(18,8)$ \\
Oftalmológico & $5(7,8)$ \\
Cardiovascular & $4(6,3)$ \\
Neurológico & $1(1,6)$ \\
Gênito-Urinário & $1(1,6)$ \\
Endócrino & $1(1,6)$ \\
Auditivo & $1(1,6)$ \\
Respiratório & $1(1,6)$ \\
Pele e tecido cutâneo & $1(1,6)$ \\
Câncer & $1(1,6)$ \\
\hline
\end{tabular}

bastante aderência com os locais que mais possuíram profissionais afastados.

Cabe ressaltar que oito das 52 readaptações descritas anteriormente representam profissionais que foram readaptados por duas vezes dentro do mesmo ano, isto pode significar que as limitações estabelecidas a priori foram insuficientes e precisaram ser revisadas a posteriori ou que o profissional teve sua condição de saúde ainda mais deteriorada e precisou sofrer novas restrições laborais.

O perfil dos profissionais readaptados foi bastante similar ao dos profissionais afastados, ou seja, a maioria era do sexo feminino (79,5\%), possuía entre 46 e 55 anos $(70,4 \%)$ e exercia o cargo de técnico de enfermagem (79,5\%), o que pode indicar uma redução progressiva da capacidade laboral dos trabalhadores em virtude do adoecimento, tornando necessário o processo de readaptação funcional para tornar o seu trabalho aderente à sua restrição de saúde. Para confirmação de tal afirmação, seriam necessários mais estudos de causa-efeito.

Entre os participantes que sofreram readaptação funcional $9(20,4 \%)$ eram enfermeiros $(20,4 \%)$. O enfermeiro ocupa posições de liderança na equipe de enfermagem. A característica gerencial do enfermeiro, ao mesmo tempo em que o afasta do contato direto com o paciente, o expõe a uma elevada carga de estresse, por ser responsável pela realização do seu trabalho e a supervisão do trabalho dos outros. Em decorrência desta situação, é sabido que os enfermeiros acabam por evitar o afastamento, mesmo doentes, em razão da responsabilidade para com a sua equipe ${ }^{7}$.

Os dois principais motivos encontrados para a readaptação funcional foram os mesmos que motivaram o afastamento dos outros profissionais estudados, isto é, os problemas "osteomusculares" apareceram com $56,2 \%$ e problemas de "saúde mental" com $18,7 \%$. Os problemas osteomusculares têm grande relevância no processo de readaptação funcional. Um estudo apontou que a dor corporal foi um dos domínios mais referidos por profissionais de enfermagem readaptados, pois ela é incapacitante, além de alterar a concentração e atenção durante a realização de tarefas laborais ${ }^{33}$. 
A precarização multidimensional do ambiente laboral, como a carência de recursos materiais e humanos, por exemplo, que impelem no trabalhador a auto aceleração para suprir esta carência, e a obrigatoriedade da polivalência para que possa realizar múltiplas tarefas ao mesmo tempo potencializam o desgaste ${ }^{29-34}$.

\section{CONCLUSÃo}

A maior parte dos profissionais foram afastados por fatores que influenciam o estado de saúde e o contato com serviços de saúde, com destaque para doenças do sistema osteomuscular e do tecido conjuntivo e transtornos mentais e comportamentais. $O$ número total de dias afastados, que foi de 29.931 dias, representa mais de 82 anos de trabalho perdidos. Em relação aos profissionais readaptados, encontrou-se como principais causas de readaptação acometimentos osteomusculares e de saúde mental. Apesar de sua relevância, a readaptação funcional dos profissionais de enfermagem ainda é um tema pouco discutido e escasso em publicações. Tal situação dificulta o entendimento mais claro dos motivos que resultam na limitação da capacidade laboral dos profissionais, fazendo-se necessário que mais estudos sejam realizados nessa área.

\section{REFERÊNCIAS}

1. Alves AP, Pedrosa LAK, Coimbra MAR, Miranzi MAS, Hass VJ. Prevalência de transtornos mentais comuns entre profissionais de saúde. Rev. enferm. UERJ. 2015; 23(1):64-9. Doi: http:// dx.doi.org/10.12957/reuerj.2015.8150

2. Felli VEA. Condições de trabalho de enfermagem e adoecimento: motivos para a redução da jornada de trabalho para 30 horas. Enferm. foco[Internet]. 2012 [citado 2016 Dez 2015]; 3(4):178-81. Disponível em: http://revista.portalcofen.gov.br/ index.php/enfermagem/article/view/379/170

3. Gonçalves FGA, Souza NVDO, Pires AS, Santos DM, D’Oliveira CAFB, Ribeiro LV. Neoliberal model and its implications for the health of nursing workers. Rev. enferm. UERJ [Internet]. 2014 [cited 2016 May 12]; 22(4):519-25. Available from: http:// www.e-publicacoes.uerj.br/index.php/enfermagemuerj/article/view/15395/11646

4. Mininel VA, Felli VEA, Silva EJ, Torri Z, Abreu AP, Branco MTA. Workloads, strain processes and sickness absenteeism in nursing. Rev. latinoam. Enferm. 2013; 21(6):1290-97. http:// dx.doi.org/10.1590/0104-1169.2992.2366

5. Marques DO, Pereira MS, Souza ACS, Vila VSC, Almeida CCOF, Oliveira EC. Absenteeism - illness of the nursing staff of a university hospital. Rev. bras. enferm. 2015; 68(5):876-82. http://dx.doi.org/10.1590/0034-7167.2015680516i

6. Silva AM, Guimarães LAM. Occupational stress and quality of life in nursing. Paidéia. 2016; 26(63):63-70. http://dx.doi. org/10.1590/1982-43272663201608

7. Ferreira RC, Griep RH, Fonseca MJM, Rotenberg L. A multifactorial approach to sickness absenteeism among nursing staff. Rev. saúde pública. 2012; 46(2):259-68. http://dx.doi. org/10.1590/S0034-89102012005000018

8. Faria JIL, Kurcgant P. Nursing professionals' sick leave: characteristics and actions adopted by nurse managers. Cogitare enferm. 2014; 19(2):224-31. http://dx.doi.org/10.5380/ ce.v19i2.34334

9. Dutra FCMS, Costa LC, Sampaio RF. The influence of medical work leaves in the perception of health and quality of life of adult individuals. Fisioter Pesqui. 2016; 23(1):98-104. http:// dx.doi.org/10.1590/1809-2950/14900923012016

10. Rouquayrol MZ, Gurgel M. Epidemiologia e Saúde. 7. ed. Rio de Janeiro: Medbook, 2013.

11. Fischborn AF, Viegas MF. A atividade dos trabalhadores de enfermagem numa unidade hospitalar: entre normas e renormalizações. Trab educ saúde. 2015; 13(3):657-74. http://dx.doi. org/10.1590/1981-7746-sip00060.

12.Gutierrez BAO, Ciampone MHT. Profissionais de enfermagem frente ao processo de morte em unidades de terapia intensiva. Acta Paul. Enferm. 2006; 19(4):456-61. http://dx.doi. org/10.1590/S0103-21002006000400015.

13.Silva AM, Guimarães LAM. Occupational stress and quality of life in nursing. Paidéia. 2016; 26(63):63-70. http://dx.doi. org/10.1590/1982-43272663201608.

14. Gonçalves FGAG, Souza NVDO, Pires AS, Santos DM, D'Oliveira CAFB, Ribeiro LV. Modelo neoliberal e suas implicações para a saúde do trabalhador de enfermagem. Rev. enferm. UERJ [Internet]. 2014 [citado 29 mar 2016]; 22(4):519-25. Disponível em: http://www.facenf.uerj.br/v22n4/v22n4a14.pdf\&gt.

15.Shoji S, Souza NVDO, Farias SNP. Impacto do ambiente laboral no processo saúde doença dos trabalhadores de enfermagem de uma unidade ambulatorial especializada. REME rev. min. enferm. 2015; 19(1): 43-8. http://www.dx.doi. org/10.5935/1415-2762.20150004.

16.Mininel VA, Baptista PCP, Felli VEA. Psychic workloads and strain processes in nursing workers of Brazilian university hospitals. Rev. latinoam. enferm. 2011; 19(2): 340-7. http:// dx.doi.org/10.1590/S0104-11692011000200016.

17. Malaguti SE, Hayashida M, Canini SRMS, Gir E. Enfermeiros com cargos de chefia e medidaspreventivas à exposição ocupacional: facilidades e barreiras. Rev. Esc. Enferm. USP. 2008; 42(3):496-503. http://dx.doi.org/10.1590/S0080-62342008000300012.

18. Sass O. Sobre os conceitos de censo e amostragem em educação, no Brasil. Estatística e Sociedade [Online]. 2012[Acesso em 28 Ago 2018]; 2:128-41. Disponível em: http://seer.ufrgs.br/index.php/estatisticaesociedade/article/download/34902/23645. 19. Machado MH, Filho WA, Lacerda WF, Oliveira E, Lemos W, Wermelinger $\mathrm{M}$, et al. Características Gerais da Enfermagem: o perfil sociodemográfico. Enferm. foco. 2016; 7:9-14. https:// doi.org/10.21675/2357-707X.2016.v7.nESP.686.

20.Santana LL, Miranda FMAM, Karino ME, Baptista PCP, Felli VEA, Sarquis LMM. Description of workloads and fatigue experienced among health workers in a teaching hospital. Rev. gaúch. enferm. 2013; 34(1):64-70. DOI: http://dx.doi. org/10.1590/S1983-14472013000100008.

21.Bernardes CL, Vasconcelos LHS, Silva SM, Baptista PCP, Felli VEA, Pustiglione Met al.Health problems of nursing workers in a public educational institution. Rev. Esc. Enferm. USP. 2014; 48(40):677-83. DOI: http://dx.doi.org/10.1590/S0080623420140000400015.

22.22.Guimarães ALO, Felli VEA. Notification of health problems among nursing workers in university hospitals. Rev. bras. enferm. 2016; 69(3):507-14. DOI: http://dx.doi. org/10.1590/0034-7167.2016690313i

23.Rodrigues BC, Lima MF, Maschio Neto B, Oliveira GL, Corrêa ACP, Higarashi IH. Being a mother and a nurse: issues about gender and overlapping social roles. Rev. Rene. 2017; 18(1):91-8. DOI: http://www.dx.doi.org/10.15253/21756783.2017000100013

24.Araújo GS, Sampaio AS, Santos EM, Barreto SMG, Almeida NJV, Santos MLD. Profile of nursing professionals assisted by a multidisciplinary mental health team. Rev. Rene. 2014; 15(2):257-63. DOI: http://www.dx.doi.org/10.15253/21756783.2014000200010

25. Rodrigues EP, Rodrigues US, Oliveira LMM, Laudano RCS, Nasci- 
mento Sobrinho, CL. Prevalência de transtornos mentais comuns em trabalhadores de enfermagem em um hospital da Bahia. Rev. bras. enferm. 2014; 67(2):296-301. DOI; I 10.5935/0034-7167.20140040. 26.Silva JLL, Teixeira LR, Soares RS, Costa FS, Aranha JDS, Teixeira ER. Estrés y factores psicosociales en el trabajo de enfermeros intensivistas. Enferm. Glob. 2017; 16(48):80-120. Doi: http://dx.doi.org/10.6018/eglobal.16.4.249321.

27. Karino ME Felli VEA, Sarquis LMM, Santana LL, Silva SR, Teixeira RC. Workloads and strain processes of nursing workers at teaching hospital. Ciênc. cuid. saúde. 2015; 14(2):1011-8. DOI: http://www.dx.doi.org/10.4025/cienccuidsaude.v14i2.21603 28. Felli VEA. Condições de trabalho de enfermagem e adoecimento: motivos para a redução da jornada de trabalho para 30 horas. Enferm. foco [Internet]. 2012 [citado 2016 Dez. 10]; 3(4):178-81. Disponível em: http://revista.portalcofen.gov.br/ index.php/enfermagem/article/view/379/170

29. Yasobant S; Rajkumar P. Work-related musculoskeletal disorders among health care professionals: A cross-sectional assessment of risk factors in a tertiary hospital. Indian J Occup Environ Med. 2014; 18(2):75-8. DOI: http://dx.doi. org/10.4103/0019-5278.146896
30. Falavigna A, Carlotto MS. Tendência temporal de afastamento do trabalho por transtornos mentais e comportamentais em enfermeiros (1998-2008). Rev psicol organ Trab [Internet]. 2013[citado 2016 Dez 15]; 13(3):363-71. Disponível em: DOI: http://pepsic.bvsalud.org/pdf/rpot/v13n3/v13n3a12.pdf 31. Rocha SH, Bussinguer ECA. A invisibilidade das doenças mentais ocupacionais no mundo contemporâneo do trabalho. Pensar [Internet]. 2016[citado 2016 Jun. 10]; 21(3):1104-22. Disponível em: http://periodicos.unifor.br/rpen/article/download/4470/pdf_1 32. Fakih FT, Tanaka LH, Carmagnani MIS. Nursing staff absences in the emergency room of a university hospital. Acta paul enferm. 2012; 25(3):378-85. DOI: http://dx.doi.org/10.1590/ S0103-21002012000300010.

33.Cacciari P, Haddad MCL, Vannuchi MTO, Dalmas JC. Health status of nursing workers in functional retraining and readaptation. Rev bras enferm. 2013; 66(6):860-5. DOI: http://dx.doi. org/10.1590/S0034-71672013000600008.

34.Souza NVDO, Gonçalves FGA, Pires AS, David HMSL. Influência do neoliberalismo na organização e processo de trabalho hospitalar de enfermagem. Rev bras enferm. 2017, 70(5):961-9. DOI: http://dx.doi.org/10.1590/0034-7167-2016-0092. 